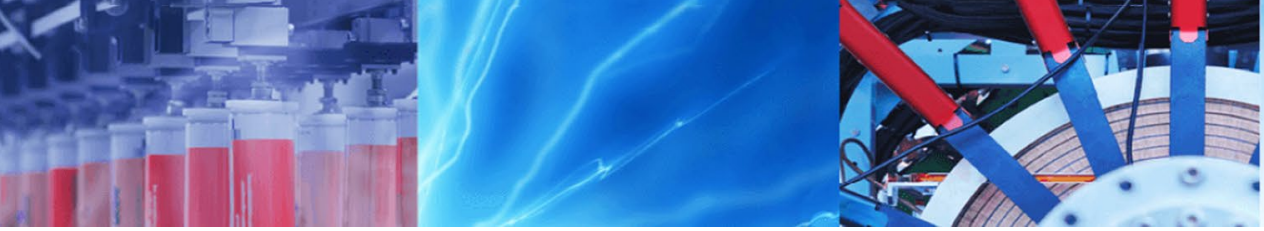

Research Article

\title{
A multilateral analysis of slope failure due to liquefaction-induced lateral deformation using shaking table tests
}

\author{
Mohammad Sadegh Maghsoudi ${ }^{1} \cdot$ Reza Jamshidi Chenari $^{2}$ D $\cdot$ Farhang Farrokhi ${ }^{1}$
}

Received: 21 May 2020 / Accepted: 20 July 2020 / Published online: 27 July 2020

(c) Springer Nature Switzerland AG 2020

\begin{abstract}
Liquefaction-induced lateral displacement can cause substantial deformations and trigger failure in the saturated sandy slopes. This study elaborates on the results of a shaking table experiment performed on a saturated sandy slope model. A transparent Plexiglass container was used to allow visualization of the time-dependent ground displacements at different grid points. Using colored sand grid along with the video-tracking analysis technique provided an excellent opportunity for instant monitoring of the model deformation in both the time and space domains. Furthermore, the pore pressure buildup and dissipation in the soil medium and acceleration time histories of the model were recorded and analyzed. Detailed and comprehensive analyses of the high-resolution recorded data streams resulted in the determination of the onset of liquefaction and the induced subsidence, acceleration response, deformation behavior, and shear strain progress through time and depth at different slope sections. It was also found that the shear strain rate was different in the on-slope and free field locations, and its value was proportional to the liquefaction duration. It was also observed that the variation of the shear strain rate at different depths in the free field led to a localized sliding event.
\end{abstract}

Keywords Shaking table test $\cdot$ Liquefaction $\cdot$ Lateral spreading $\cdot$ Anzali sand $\cdot$ Sloping ground

\section{Introduction}

Liquefaction is a phenomenon during which a saturated soil, mainly sand, significantly loses its shear strength when subjected to the cyclic shear loading, commonly induced by major earthquakes, and flows like a liquid [1]. Earthquake-induced liquefaction can lead to large settlements and localized failure modes (i.e., sliding surfaces) and causes instability in the geo-structures.

During liquefaction, soil undergoes various types of deformation depending on its topography and in situ conditions. In sub-horizontal ground conditions, the soil deposit may settle following the liquefaction-induced bearing capacity loss. Moreover, if there exist non-liquefiable layers above a liquefied layer, they may collide during ground oscillations [2]. In gently sloped grounds (typically grounds with slopes less than $5 \%$ ), the surficial soil layers may slightly move down the slope. In the cases where there are topographic irregularities (i.e., channel and lake banks) in the liquefied area, soil tends to displace toward a free-face (or abrupt topography change), along a critical shear zone formed by liquefaction [3].

Since the California earthquake in 1971, where the Lower San Fernando Dam collapsed [4], and until the recent 2010 Maule earthquake in Chile and 2011 Tohoku earthquake in Japan $[5,6]$, several cases of liquefactioninduced ground catastrophes in the earth embankments have occurred [7-9]. Some researchers have reported copious case histories of liquefaction-induced slope failures in

$\triangle$ Reza Jamshidi Chenari, Jamshidi_reza@yahoo.com; Mohammad Sadegh Maghsoudi, maghsoudi@znu.ac.ir; Farhang Farrokhi, Farhang.Farrokhi@znu.ac.ir| 'Department of Civil and Environmental Engineering, Faculty of Engineering, University of Zanjan, Zanjan, Iran. ${ }^{2}$ Department of Civil Engineering, Faculty of Engineering, University of Guilan, P.O. 3756 Rasht, Guilan, Iran. 
the embankments, earth dams, and natural slopes during earthquakes $[10,11]$.

The excessive displacements created by liquefaction in susceptible topographic conditions have brought about many researchers to keep studying in this field. The very seminal study of Youd [3] classified the liquefaction-induced lateral displacement as gently sloping and free-face conditions. Physical modeling of a soil slope was then performed using centrifuge tests by TaboadaUrtuzuastegui, and Dobry [12], and the influence of ground slope, peak input acceleration, and excitation frequency parameters on the lateral ground displacement was thoroughly investigated [12]. In order to understand the response of the liquefied sandy slopes after initial liquefaction, Taboada-Urtuzuastegui et al. [13] carried out a series of centrifuge tests. They emphasized on the dilative behavior of the soil during liquefaction and its impact on the subsequent lateral displacements. The mechanisms of seismically induced permanent deformations in slopes were investigated by Wartman et al. [14]. They examined the results of $1 \mathrm{~g}$ shaking table tests on slope models having different layering conditions and elaborated on the displacement distribution in the soil models [14]. This was followed by Dobry et al. [15] by carrying out full-scale shaking table tests. In their study, initiation and accumulation of lateral spreading at various depths and their relation to the excess pore water pressure and the soil shear behavior during liquefaction were discussed. The liquefaction-induced deformation of an embankment located on a non-homogeneous soil deposit was investigated by Maharjan and Takahashi [16]. They focused on the influence of various foundation conditions on the embankment deformations. In a recent study, Rapti et al. [17] studied the earthquake-induced liquefaction failure modes of a model founded on liquefiable soil substratum through the dynamic nonlinear finite element analysis.

The current study summarizes the results of the shaking table tests on a sloping ground to study the liquefaction consequences of sandy slopes during and after shaking. This is done using the video analysis technique, which has the advantage of continuously monitoring the deformation of the model, allowing to survey the behavior of the model at any desired point and at any time.

\section{Test equipment}

\subsection{Shaking table}

The experimental results presented in this study are the outputs of a physical model experiment on the shaking table at the soil laboratory of University of Guilan. This shaking table, being a computer-controlled servo-electric device and capable of creating and simulating the horizontal seismic movements, consists of several components such as the central loading platform, the loading frame, the digital controller, the servo-electric actuator, and the interface software. Frictionless movement of the shaking table was guaranteed through the provision of a pair of linear bearings on the loading structure with a payload of $500 \mathrm{~kg}$. In addition, a digitally controlled servo-electric actuator with a maximum displacement of $150 \mathrm{~mm}$ and of $4.5 \mathrm{~kW}$ power provided sinusoidal waves with the frequency ranging from 0.01 to $200 \mathrm{~Hz}$. The loading platform was designed with a natural frequency being above $50 \mathrm{~Hz}$, large enough to ensure prevention of the resonance occurrence under seismic excitations. This shaking table also had a foundation of $3 \times 2.5 \times 2$ meters and a weight of approximately 35 tons, assuring no overall movement of shaking table.

For continuous measuring of the horizontal displacement of the model box, the linear variable differential transformer (LVDT) was utilized in the base of the shaking table. The maximum measurement range of the LVDT was $100 \mathrm{~mm}$, with a resolution of $0.01 \mathrm{~mm}$.

\subsection{Model container}

The physical model was constructed and tested in a transparent Plexiglass box with internal dimensions of $900 \times 500 \times 500 \mathrm{~mm}$ in length, width, and height, respectively. The use of Plexiglass made it possible to monitor and track the model deformations and failure progress. For this purpose, a $10-\mathrm{cm}$ square grid was marked on one side of the transparent box. In order to prevent the wall deflection, a 15-mm Plexiglass sheet was opted to fabricate the container box. The walls of the box were rigidly connected together with special glue. Moreover, the screws were sealed accurately in the secure intervals and all the connection sides to prevent water leakage. Although the rigidity of the box caused relatively different soil behavior in the vicinity of the walls compared to the other points, it was believed that "reasonably correct" results can be obtained for the soils away from the walls which are somehow not affected by the rigid boundaries [18] and for numerical modeling purposes, the rigid walls can be considered as certain boundary conditions.

At the bottom of the box, a chamber was designed for the uniform water entry into the model and sample saturation from the bottom to the top. In fact, the water entered the chamber through the inlet valve and then penetrated into the model uniformly. For this purpose, a number of incompressible columns with a height of $5 \mathrm{~cm}$ were placed and fixed to the bottom of the box. Then, a composite plate, on which tiny holes were created at very close distances, was firmly placed on these columns. These 
closely spaced holes caused a more uniform water entry and saturation of the sample. Next, a stainless-steel No. 325 filter mesh (with $45-\mu$ m openings) was placed on top of the composite plate to prevent soil particles from washing into the tiny holes and soil clogging to happen.

\subsection{Rigid frame}

In order to prevent damage to the Plexiglass box and securely connecting the model container to the shaking table, a rigid steel frame was designed, and the model container was placed inside it. The frame consisted of two parts: one part to hold the model container and the other part to fix it to the shaking table. The two-piece frame allowed adjustment of the model's slope to render the designated inclinations. This was done using some long screws on one side and a hinge on the other side of the frame.

\subsection{Pore pressure transducers and data logging}

Two pore pressure transducers mounted and fixed on one side of the box measured the excess pore water pressure (EPWP) generation and dissipation during the shaking table test. Each of these transducers measured the pore water pressure through a rigid steel tube extending up to half the width of the box. These two steel tubes were fixed at the height of one-third and two-thirds of the total height of the box. Before the experiment, the calibration of the pore pressure transducers was accomplished by piezometers. A dual-channel data logger was also used to record the variation of the excess pore water pressure during and after shaking at a specified time interval, typically
$0.01 \mathrm{~s}$. Figure 1 shows the shaking table and test equipment facility.

\subsection{High-speed digital camera}

In order to measure the displacement and deformation of the model during the test, a high-speed video camera was placed at a fixed point. With this camera, the movement of the soil body was recorded at a high-speed rate at any moment, and then using the motion tracker software, it was possible to analyze frame-by-frame the lateral spreading and slope failure.

\section{Materials and methods}

\subsection{Soil properties}

The soil used for physical modeling in this study was Anzali sand, a typical soil founded in a coastal area located on the southern side of the Caspian Sea in northern Iran. The Anzali sand is mostly fine-grained, saturated, and has some silt content. The characterization of this soil indicates that the soil is susceptible to liquefaction during earthquakes [19]. Figure 2 shows the grain size distribution [20] of the Anzali sand along with the distribution range of susceptible liquefiable soils introduced by Tsuchida [21]. As evident from Fig. 2, the gradation curve of the Anzali sand lies between the Tsuchida's specified range, indicating the high liquefaction potential of this soil.

The index properties of the Anzali sand are also listed in Table 1. Based on the laboratory test results, the soil is a poorly graded sand and classified as SP, according to the unified classification system [22].

Fig. 1 Shaking table and soil container used in this study

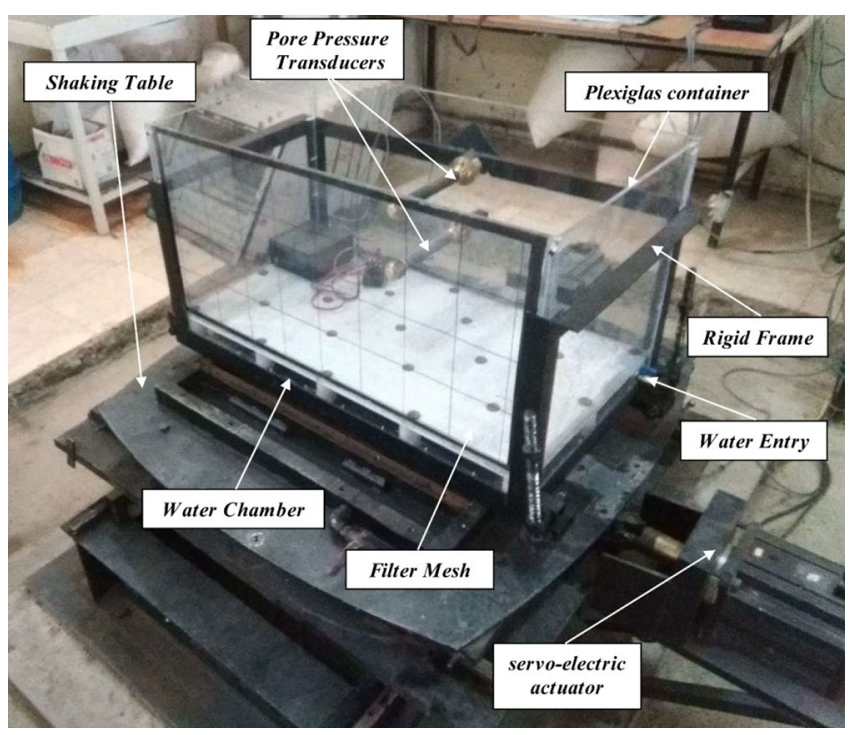




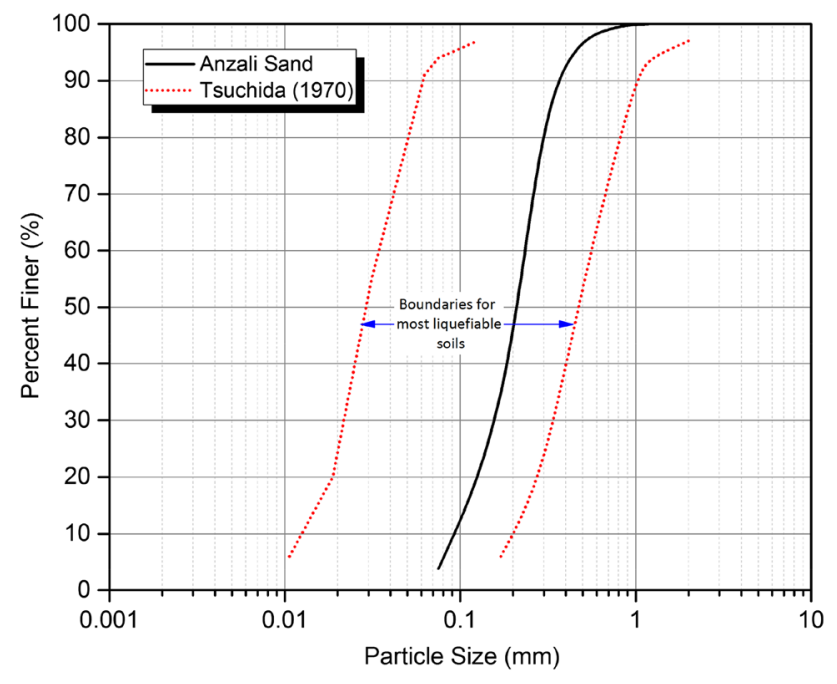

Fig. 2 Grain size distribution curve for the Anzali sand

Table 1 Anzali sand properties from laboratory test results

\begin{tabular}{ll}
\hline Property & Value \\
\hline USCS & $\mathrm{SP}$ \\
$D_{10}(\mathrm{~mm})$ & 0.1 \\
$D_{50}(\mathrm{~mm})$ & 0.34 \\
$C_{u}$ & 1.8 \\
$C_{c}$ & 0.9 \\
$G_{s}$ & 2.67 \\
$e_{\min }$ & 0.55 \\
$e_{\max }$ & 0.88 \\
\hline
\end{tabular}

\subsection{Model preparation and test procedure}

Soil preparation is one of the most critical and influential stages in soils testing, especially in the large-scale physical model experiments, such as shaking table and centrifuge tests. There are mainly three methods of sample preparation, including dry deposition, wet tamping, and water sedimentation methods. Each of these three methods can produce different results under the same loading conditions [23]. In this study, the water sedimentation method was utilized to construct the slope model. For this purpose, oven-dried sand was continuously poured into the water using a hopper, which was moved manually along the length of the box at a constant height from the surface of the water to achieve a uniform model of sand with the desired relative density. In order to control the relative density of the sample, the height of the sand pluviation was adjusted according to the preliminary calibration experiments. Having weighted the poured dry sand, the dimensions of the model (as in Fig. 3), and the use of the universal weight-volume relationships, the relative density of the model was measured to be about $38 \%$.

The vertical colored lines were made by thin aluminum channels, mounted correctly aligned with lines drawn on the box wall before sample preparation. These channels were filled with colored sand similar to the sand used for modeling. Horizontal color lines were created in the predetermined locations. After completing the model and reaching the specified levels, the channels were slowly and gradually withdrawn from the soil model, and the colored sand grid was therefore formed.

After preparing the model, the digital camera was mounted to watch the side of the box meshed with colored sand in order to record the process of testing and deformations of the model. Harmonic displacements were then applied to the model with a predetermined frequency, amplitude, and shaking duration. The image recording process and excess pore water pressure measuring were continued well after the end of the shaking.
Fig. 3 Configuration of the tested slope model

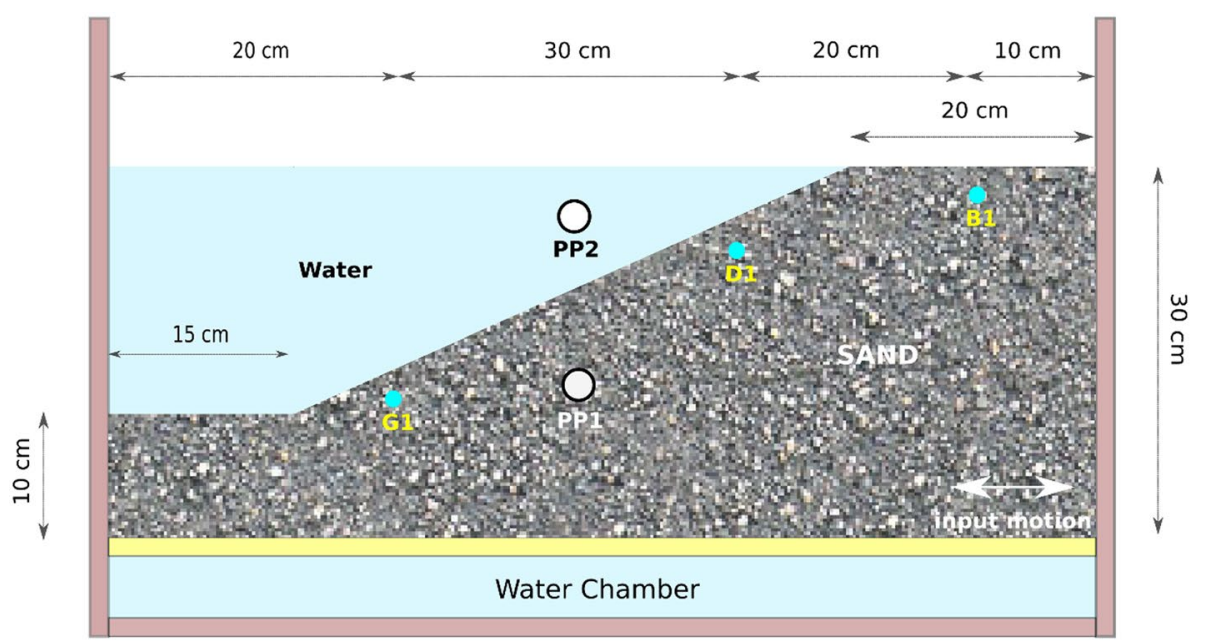




\section{Test results and analyses}

The results presented in this section are based on the frame-by-frame analysis of the images captured by a high-speed digital camera during the test. This was carried out by video-tracking software that was able to identify marked points and track them over time. Using this technique, the time histories of soil displacement could be obtained for marked points along with both length and depth directions. Subsequently, other parameters such as speed, acceleration, and shear strain could also be derived using this technique.

A schematic representation of the model configuration is shown in Fig. 3. For the analysis of the seismic behavior of the model during shaking, three points of $B_{1}$, $D_{1}$, and $G_{1}$ were selected according to the position of the marked lines. These three points were located near the slope, on the slope, and on the toe of the slope, respectively. In the following subsections, different results of the experiment are presented separately.

\subsection{Accelerations and soil responses}

The input motion applied to the model was a harmonic excitation with an amplitude of $2 \mathrm{~mm}$, the frequency of $6 \mathrm{~Hz}$, and the duration of $20 \mathrm{~s}$. The selection and use of the above parameters were made after performing some preliminary tests on the model before the main tests. To this end, by observing the onset of liquefaction in preliminary experiments, the duration of the input motion was selected so that the soil behavior can be evaluated sufficiently after the onset of liquefaction during and after shock. Furthermore, the frequency and amplitude of the excitation were chosen so that the model did not undergo significant deformation before the initiation of liquefaction (due to its low density and looseness) and the slope deformation behavior during liquefaction can be examined.

Figure 4a shows the displacement time history of the input motion. Input acceleration time history was consequently derived by double differentiation of the input motion. This is shown in Fig. 4b. From this figure, it can be seen that the maximum acceleration $\left(a_{\max }\right)$ of the input motion is about $0.65 \mathrm{~g}$.

The acceleration time histories of the near-slope, onslope, and toe are also provided in Fig. 4c-e, respectively. These acceleration time histories have been obtained from the analysis of the displacement time histories of these three points. A significant overall attenuation in the maximum acceleration $\left(a_{\max }\right)$ for all three points is evident compared to the maximum acceleration of the base. This could be due to the partial dilation of the soil during the liquefaction phenomenon, and pore pressure-related loss of soil stiffness and shear strength [16]. Another important note is the significant non-uniform spikes in negative acceleration, especially for on-slope and toe, about $5 \mathrm{~s}$ after the start of the shaking. Some of the previous researchers also observed this event in their studies $[13,24,25]$. These spikes are attributed to a strength increase in the saturated sand due to its "dilative-strain response" in a part of the cycle [12].

Figure 5 shows the Fourier amplitude of the input acceleration along with the near-slope, on-slope, and toe acceleration Fourier spectra. As expected, predominant frequency is about $5.5 \mathrm{~Hz}$ (similar to the input frequency of $6 \mathrm{~Hz}$ ), and unlike the major earthquake spectra, there are no significant Fourier amplitudes at the other frequencies that is because of the harmonic nature of the input motion. The maximum spectral acceleration for the input motion is about $0.27 \mathrm{~g}$. For the points studied in the model, the near-slope and toe have a relatively lower value of the maximum spectral acceleration (i.e., $0.19 \mathrm{~g}$ ), and the on-slope point has approximately half of the input motion maximum spectral acceleration (i.e., $0.13 \mathrm{~g}$ ), with a predominant frequency almost the same as that of the input motion.

\subsection{Excess pore water pressure}

The pore water pressure generation and dissipation process measured by pore pressure transducers during and after shaking are shown in Fig. 6 . This figure shows an incremental and progressive excess pore water pressure buildup $(\Delta u)$ during shaking until it reached the effective stress of the soil $\left(\sigma_{v}^{\prime}\right)$ and a short time (about three seconds) after that. Figure 6 shows the effective stress of the soil, which is shown by a dashed line. The state in which the liquefaction occurs is, by definition, when $r_{u}=1$ where $r_{u}=\Delta u / \sigma_{v}^{\prime}$. As evident from the figure, the liquefaction has occurred at about $3 \mathrm{~s}$ after the start of the shaking, and it lasted for $5 \mathrm{~s}$. This time span is shown in the figure as a colored area.

After this time span, the dissipation of the pore water pressure started, while the shaking was still continued. This was due to the rapid drainage of the pore water after the soil dilation has occurred. However, the complete pore water pressure dissipation and full drainage took a longer time.

The rate of the excess pore water pressure changes against the number of cycles was determined and is depicted in Fig. 7. In this figure, the parameter $N$ represents the number of applied uniform cycles of loading and $N_{L}$ is the number of cycles required for the triggering of the liquefaction in the desired soil. The Anzali sand in 
Fig. 4 Time histories of input motion and response acceleration at different points in the model
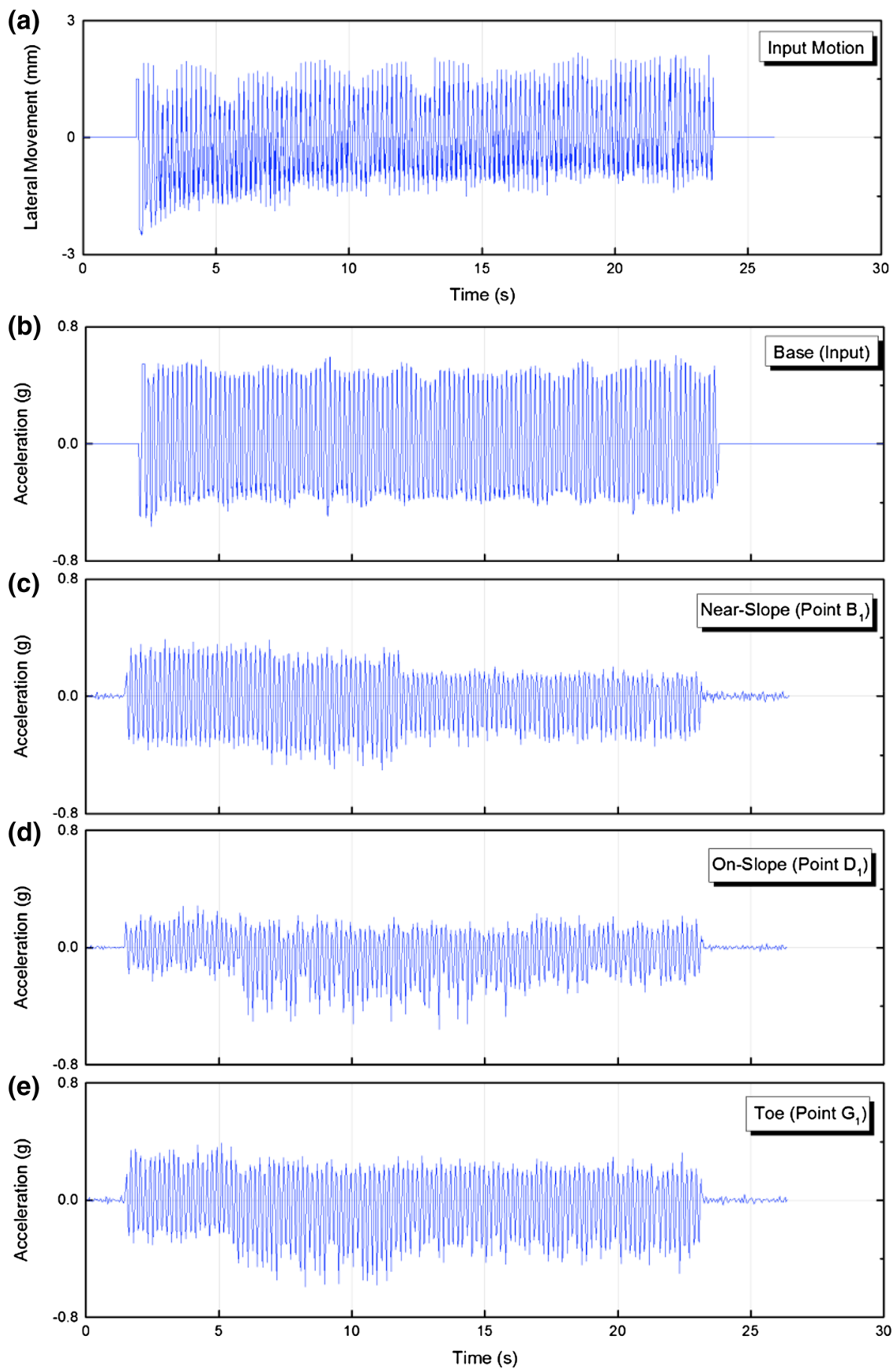

this study was liquefied after 17 cycles $\left(N_{L}=17\right)$, as can be seen from Fig. 6.

Many researchers have investigated the generation rate of the excess pore water pressure under seismic loading [26-31]. The proposed empirical curves by Seed et al. [30] and Baziar et al. [27] are depicted in Fig. 7, along with the test results in the present study. The test points are outside the range proposed by Seed et al. [30], which is probably due to the fact that their proposed curves are based on the laboratory test results on clean sand. Instead, Baziar et al. [27] made adjustments to the bounds proposed by Seed et al. [30] by introducing coefficients for considering different percentages of fine content. Since the sand used in this study contained about $5 \%$ fine content, the curves proposed by Baziar et al. [27] are re-drawn in Fig. 7, according to this fine percentage. As can be seen from this figure, the test results are well within the range mentioned above. 


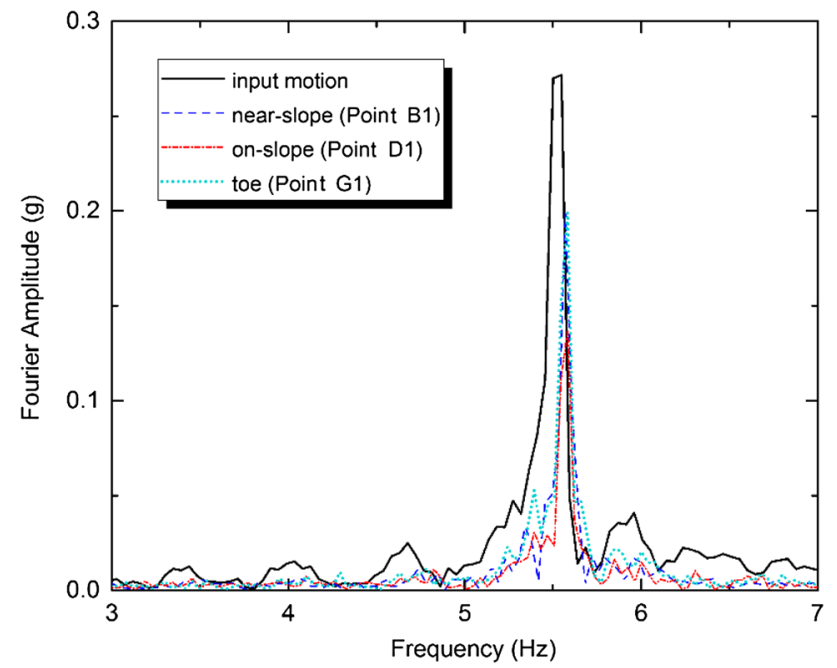

Fig. 5 Fourier spectra of input base motion and the points in the model

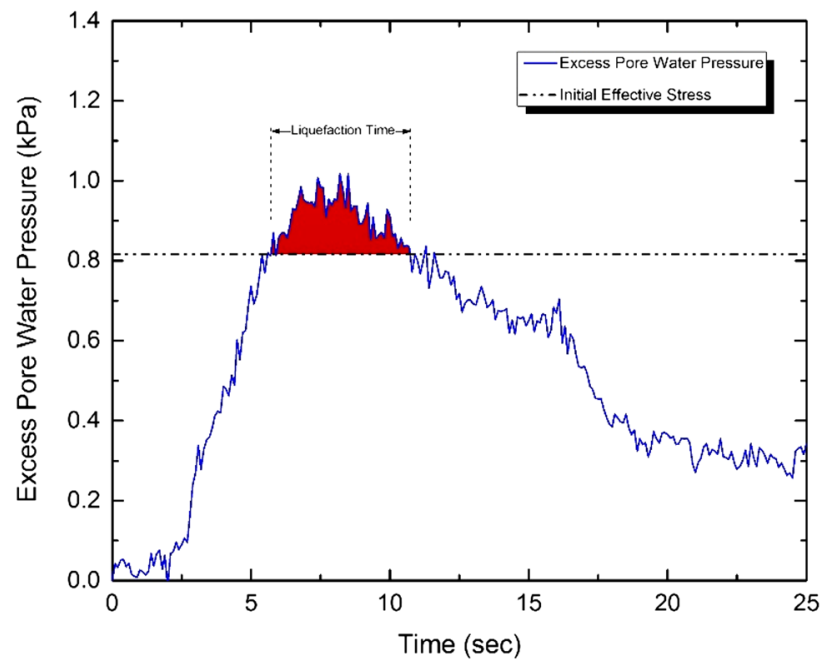

Fig. 6 Time histories of the excess pore water pressure in the model

\subsection{Lateral soil displacement and slope deformation}

Figure $8 \mathrm{a}, \mathrm{b}$ shows photographs of the constructed sloping ground model before and after shaking, respectively. The model deformation analysis in this study was carried out using motion tracking of the colored lines embedded in the model. Using this technique, it was possible to visualize the soil deformations at various points of the model $[32,33]$. Accordingly, by analyzing the ground deformation data in different locations of the model using this technique, general deformed shape, total displacement vector field, and horizontal displacement of the slope model were created at the end of the shaking, as illustrated in Fig. 9. It

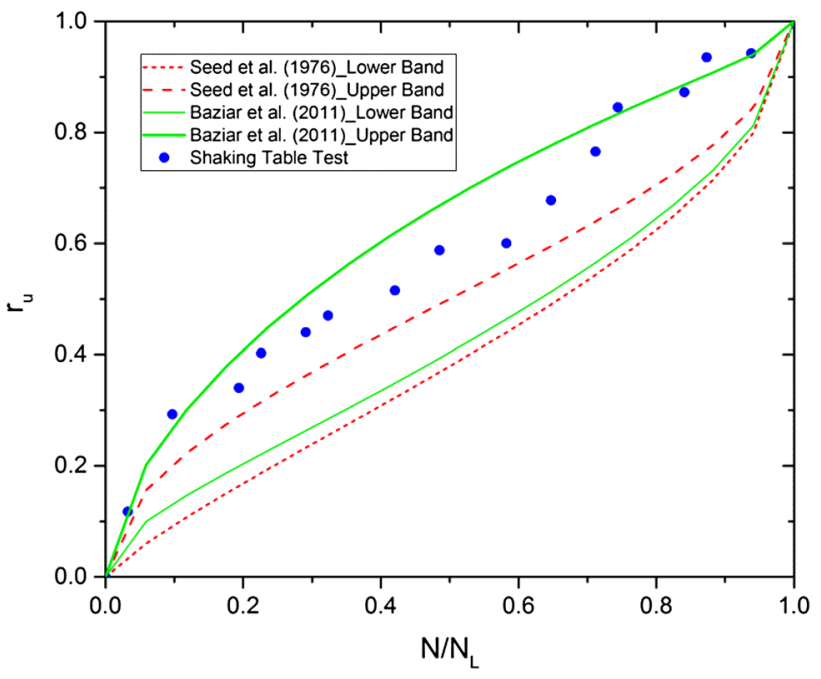

Fig. 7 Excess pore water pressure generation in the shaking table test

can be observed from the figure that the soil has generally flowed from the upstream slope toward the toe (Fig. 9a). This can be attributed both to the soil shear strength reduction through the pore water pressure buildup and to the contractive volumetric change due to the pore water pressure dissipation. On the other hand, the ground surface in the downstream was observed to rise by the amount of maximum $20 \mathrm{~mm}$ due to the accumulated sand grains (Fig. 9b).

The contour plot of the horizontal displacement for the slope model also shows that the most considerable amount of the lateral movement was related to the points located on top of the slope by a maximum value of about $30 \mathrm{~mm}$. It should be noted that the model has deformed without any distinct slip surface, as expected for a uniform sand layer without any fine-grained, very low-permeability interlayers or thin lenses [34]. The deformed area extended into the deeper part of the cross section leading to a larger area of shear deformation. The fairly more extension of the shear deformation area in depth is indeed due to the uniformity of the sand layer. Indeed, if there existed a thin fine-grained interlayer, the shear deformation regions would be limited to a narrow area above this layer [34].

Figure $10 a, b$ shows the time histories of the lateral displacements $\left(D_{h}\right)$ at different depths for near-slope and on-slope cross sections (columns B and D in Fig. 8), respectively. The curves in the charts consist of two components, including cyclic and non-cyclic (permanent) components. As can be seen from the figures, for both cross sections, the incremental lateral displacement continued to the end of the shaking at depths closer to the surface. However, for deeper points, the lateral displacement occurred in the early stages of the shaking, and almost no further 

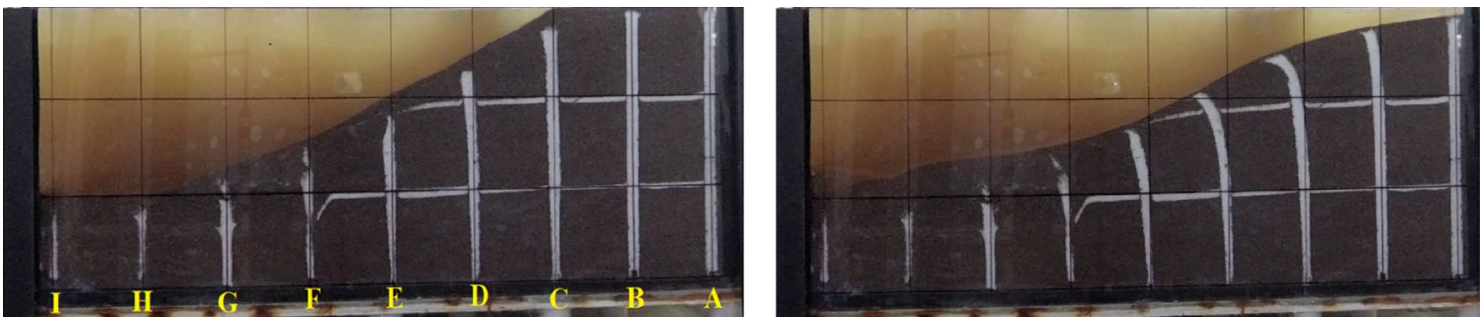

Fig. 8 Photographs of the slope model configuration before shaking (left) and at the end of the test (right)

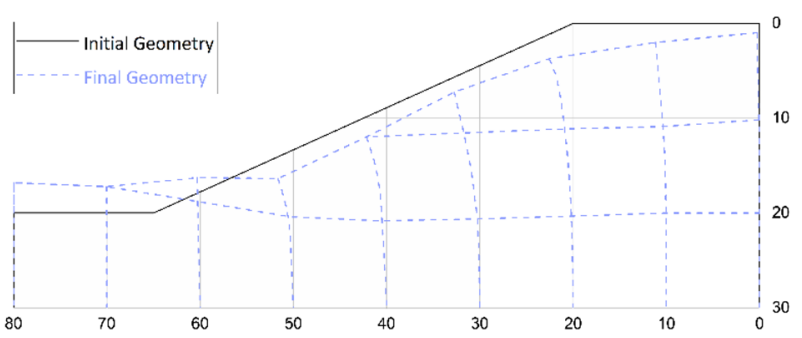

(a)

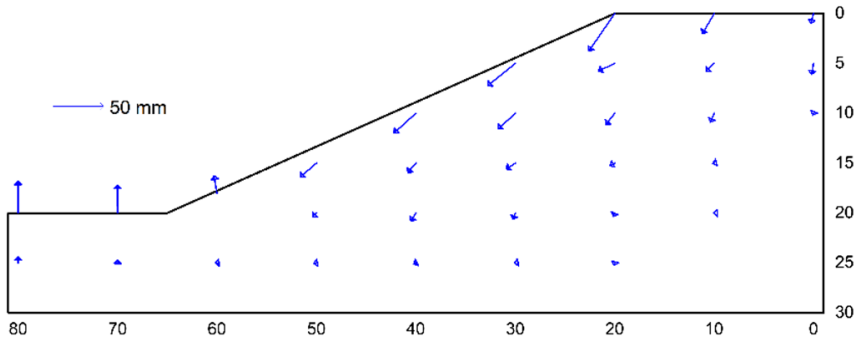

(b)

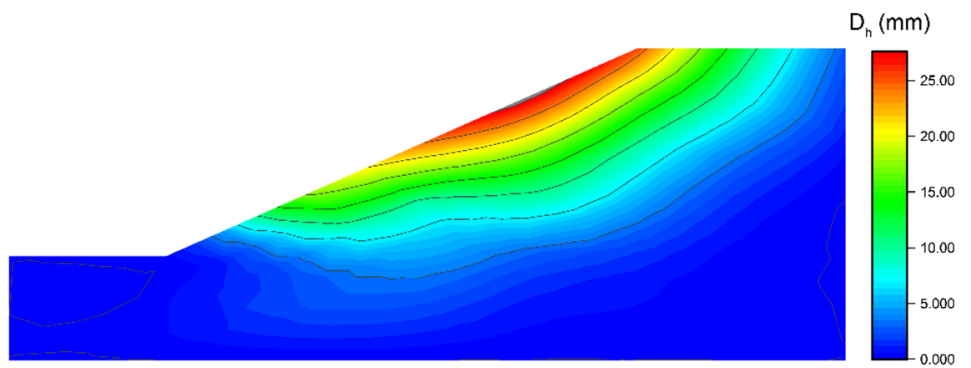

(c)

Fig. 9 Slope deformation analysis at the end of the shaking: a deformed mesh, $\mathbf{b}$ total displacement vector field, $\mathbf{c}$ horizontal displacement contour plot
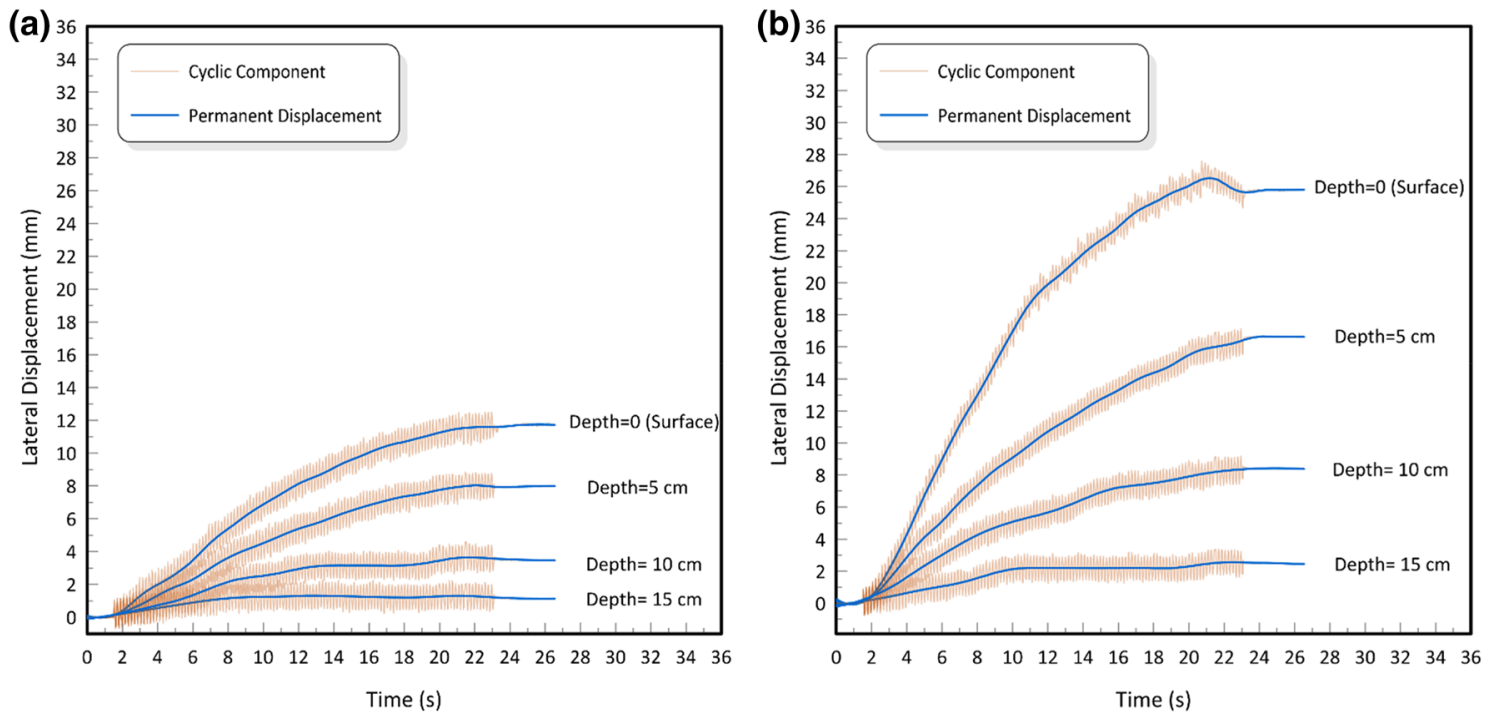

Fig. 10 Soil deformation against time for a) near-slope and b) on-slope sections 
displacement was observed afterward. It is also evident that all the ground displacements stopped as soon as the shaking ceases, and no further displacement was found in the model. This phenomenon is still related to the uniformity of the soil layer and no "water film" generation due to the lack of fine-grained interlayer [34].

\subsection{Shear strain and shear strain rate}

The profiles of the accumulated shear strain $\left(\gamma_{\max }\right)$ at the end of the test are drawn against depth in Fig. 11 for various cross sections (columns A-F in Fig. 8). This parameter is obtained by taking the first derivative of the lateral displacement relative to the depth for every point $\left(\gamma=\partial D_{h} / \partial z\right)$. Furthermore, the depth of the soil has been normalized to a dimensionless parameter in the form of $z / H$ and is shown in the right-side vertical axis

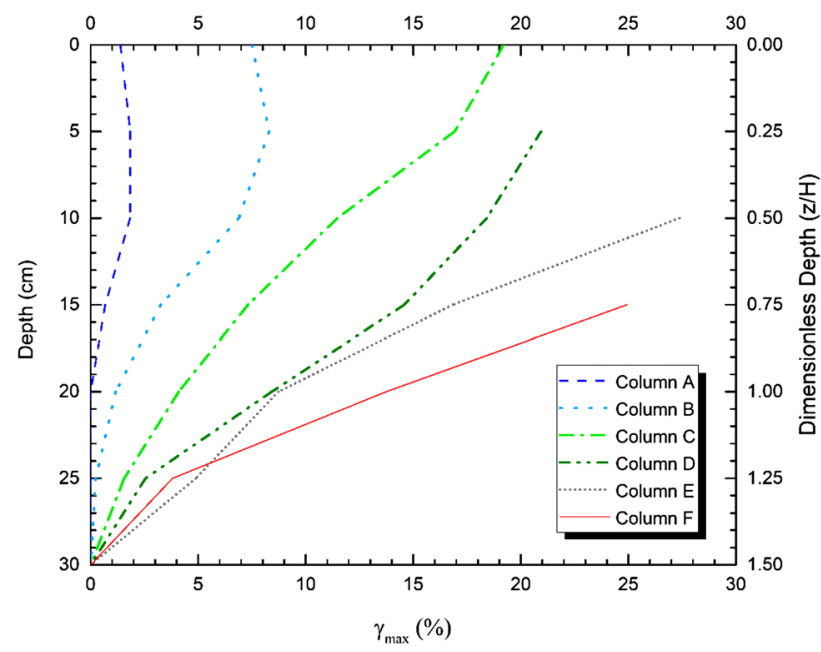

Fig. 11 Residual shear strain against depth at the end of the shaking

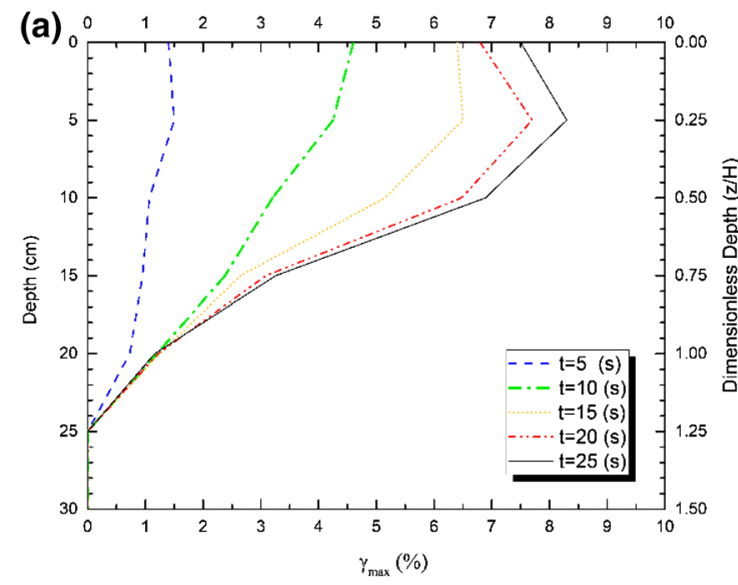

of the figure. In this expression, $\mathrm{z}$ is the distance of the desired point from the soil surface and $\mathrm{h}$ is the height of the slope (see Fig. 3). In this way, the effects of scaling can be reduced to some extent when the shear strain results are generalized to the field conditions.

As depicted in Fig. 11, for cross sections that are not on the slope (columns A and B), the maximum shear strain of the soil is not developed at the ground surface, and instead, it occurs at a depth of about $5-10 \mathrm{~cm}$ (equal to a normalized depth of $0.25-0.50$ ). However, for the rest of the locations, the shear strain profile has a distinct maximum value on the surface. This can be considered as a relatively "shallow sliding event" [15] in a limited zone at the free field beyond the slope.

In order to verify the time-dependent shear strain variation, the shear strain profiles versus the depth are depicted at different time intervals in Fig. 12a, b for the near-slope (column B) and on-slope (column D) cross sections, respectively.

The highest increase in the shear strain of the soil for both near-slope and on-slope positions has occurred between the times of 5-10 s after shaking. For a closer look at this issue, the shear strain rate $(\dot{\gamma})$ values have been calculated by taking the first derivative of the shear strain $(\gamma)$ with respect to the time $(\dot{\gamma}=\partial \gamma / \partial t)$ and plotted against the depth for both the near-slope (column B) and the onslope (column D) cross sections in Fig. 13.

By simultaneous review of the pore water pressure time history (Fig. 6) and the shear strain rate (Fig. 13), it can be observed that the significant shear strains have taken place during the period when the soil is experiencing liquefaction ( $t=5 \mathrm{~s}$ to $t=10 \mathrm{~s}$ ). During this period, the soil naturally behaved like a fluid where displacements were accentuated, and then by pore water pressure dissipation and shear strength recovery, the rate of these changes was reduced.

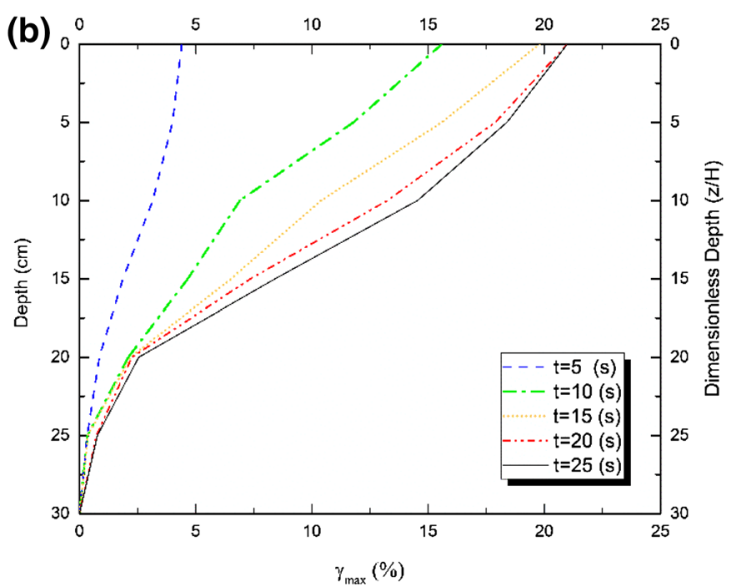

Fig. 12 Shear strain against depth at different times for $\mathbf{a}$ near-slope and $\mathbf{b}$ on-slope sections 

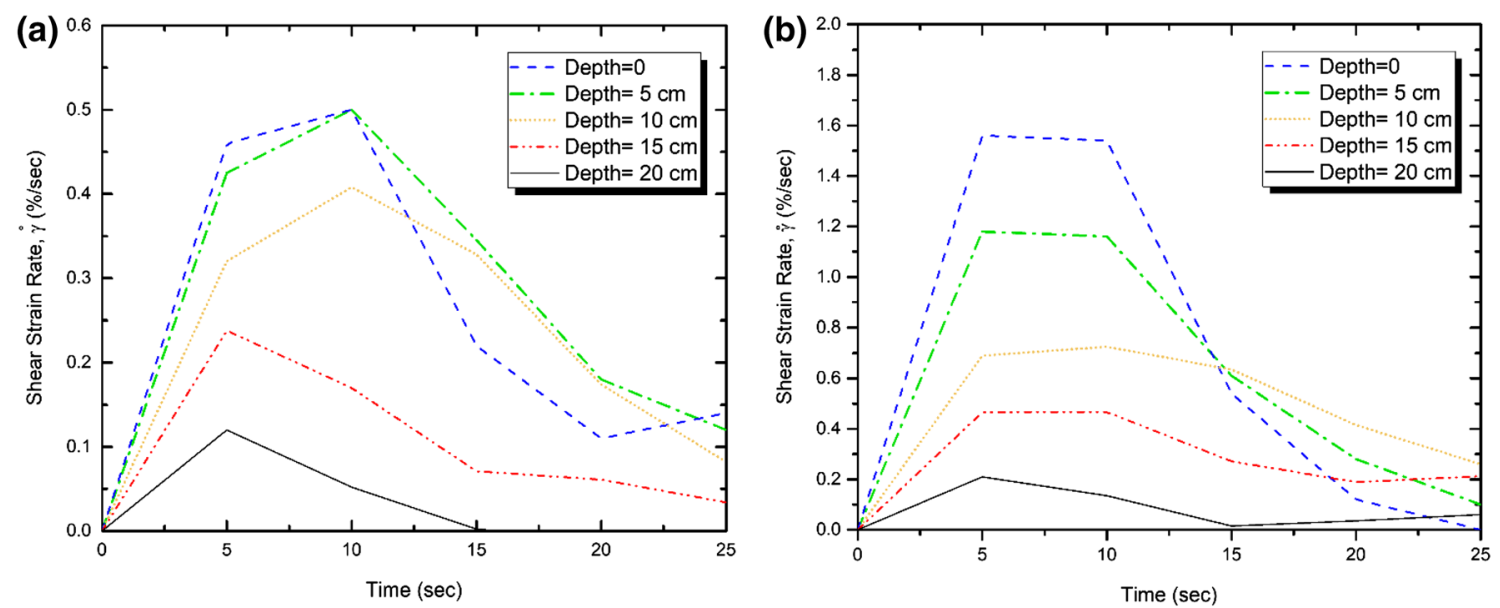

Fig. 13 Shear strain rate against time for $\mathbf{a}$ near-slope and $\mathbf{b}$ on-slope sections

In terms of the comparison of the two near-slope and on-slope cross sections, there is a slight difference in the shear strain rate variations at different depths. For the on-slope cross section (column D), a relatively consistent behavior was observed for all the depths. That implies the shear strain rate reached its maximum value at the onset of the liquefaction $(t=5 \mathrm{~s})$, remained almost constant during the liquefaction time, and gradually decreased afterward. For the near-slope cross section (column B), the maximum shear strain rate for the deeper points (the depths of 15 and $20 \mathrm{~cm}$ ) occurred at the beginning of the liquefaction $(t=5 \mathrm{~s})$, while for shallow levels, this happened later (at about $t=10 \mathrm{~s}$ ). This delay was probably responsible for the previously discussed sliding event in the free field area of the model.

\section{Summary and conclusions}

In this study, a shaking table test was conducted in order to study the behavior of a sandy slope during liquefaction. For this purpose, the Anzali sand, which is a uniform finegrained soil and has been identified as being susceptible to liquefaction, was used to construct the slope physical model in a transparent Plexiglass container, enabling the operator to observe the deformations of the model using a digital camera.

Comparison of the input acceleration data with the acceleration response of the target points (the near-slope, on-slope, and toe locations) showed a significant drop of the maximum ground acceleration $\left(a_{\max }\right)$ for all the three points due to the soil softening. Another important observation was a relatively non-uniform spike in the negative acceleration responses in all the target points.
The pore water pressure buildup and dissipation in the soil medium were measured during the test using a pore pressure transducer. These measurements showed that the liquefaction triggered about $3 \mathrm{~s}$ after the start of the shaking, and the soil endured liquefaction for $5 \mathrm{~s}$. Afterward, the pore water pressure dropped, and the liquefaction-induced subsidence occurred up to a time when the shaking was still continued. This can be attributed to the rapid drainage of the pore water after the dilation of the soil.

Liquefaction-induced lateral displacement analysis revealed an overall downward movement of the upstream slope with no clear and detectable slip surface. This phenomenon, also reported in several previous studies, was due to the uniformity of the soil and the lack of fine-grained interlayer.

The shear strain and shear strain rate of the soil during the test have also been investigated by analyzing the displacement at the target points. This analysis showed two relatively different behaviors for the free field nearslope sections and the areas located on the slope. For the on-slope cross section, the variation of the shear strain rate with time was consistent for all the points at different depths. Unlikely, for free field area, the maximum shear strain rate for deeper points occurred at the onset of the liquefaction ( $t=5 \mathrm{~s}$ ), while for locations closer to the surface, this happened with a delay. This difference is probably the cause of the localized sliding event in a limited zone in the free field.

\section{Compliance with ethical standards}

Conflict of interest On behalf of all authors, the corresponding author states that there is no conflict of interest. 


\section{References}

1. Castro G, Poulos SJ (1977) Factors affecting liquefaction and cyclic mobility. J Geotech Geoenviron Eng 103:501-516

2. National Research Council (1985) Liquefaction of soils during earthquakes. National Academies, Washington

3. Bartlett SF, Youd TL (1992) Empirical analysis of horizontal ground displacement generated by liquefaction-induced lateral spreads. Technical report NCEER-92-0021, State University of New York at Buffalo, National Center for Earthquake Engineering Research

4. Seed HB, Mori K, Chan CK (1977) Influence of seismic history on liquefaction of sands. J Geotech Geoenviron Eng 103:257-270

5. Oka F, Tsai P, Kimoto S, Kato R (2012) Damage patterns of river embankments due to the 2011 off the Pacific Coast of Tohoku Earthquake and a numerical modeling of the deformation of river embankments with a clayey subsoil layer. Soils Found 52:890-909

6. Ishikawa H, Saito K, Nakagawa K, Uzuoka R (2015) Liquefaction analysis of a damaged river levee during the 2011 Tohoku earthquake. In: Computer methods and recent advances in geomechanics: proceedings of the 14th international conference of international association for computer methods and recent advances in geomechanics, 2014 (IACMAG 2014). Taylor \& Francis Books Ltd, pp 673-677

7. Verdugo R, Sitar N, Frost JD et al (2012) Seismic performance of earth structures during the February 2010 Maule, Chile, earthquake: dams, levees, tailings dams, and retaining walls. Earthq Spectra 28:75-96

8. Okamura M, Tamamura S, Yamamoto R (2013) Seismic stability of embankments subjected to pre-deformation due to foundation consolidation. Soils Found 53:11-22

9. Ozutsumi O, Sawada S, lai S et al (2002) Effective stress analyses of liquefaction-induced deformation in river dikes. Soil Dyn Earthq Eng 22:1075-1082

10. Seed HB (1987) Design problems in soil liquefaction. J Geotech Eng 113:827-845

11. Hamada M (1993) Liquefaction-induced large ground displacement, performance of ground and soil structures during earthquakes. In: 13th international conference on soil mechanics and foundation engineering. pp 93-108

12. Taboada-Urtuzuastegui VM, Dobry R (1998) Centrifuge modeling of earthquake-induced lateral spreading in sand. J Geotech Geoenviron Eng 124:1195-1206

13. Taboada-Urtuzuastegui VM, Martinez-Ramirez G, Abdoun T (2002) Centrifuge modeling of seismic behavior of a slope in liquefiable soil. Soil Dyn Earthq Eng 22:1043-1049

14. Wartman J, Seed RB, Bray JD (2005) Shaking table modeling of seismically induced deformations in slopes. J Geotech Geoenviron Eng 131:610-622

15. Dobry R, Thevanayagam S, Medina C et al (2010) Mechanics of lateral spreading observed in a full-scale shake test. J Geotech Geoenviron Eng 137:115-129

16. Maharjan M, Takahashi A (2014) Liquefaction-induced deformation of earthen embankments on non-homogeneous soil deposits under sequential ground motions. Soil Dyn Earthq Eng 66:113-124

17. Rapti I, Lopez-Caballero F, Modaressi-Farahmand-Razavi A, Foucault A, Voldoire F (2018) Liquefaction analysis and damage evaluation of embankment-type structures. Acta Geotechnica 13(5):1041-1059

18. Whitman RV, Lambe PC (1986) Effect of boundary conditions upon centrifuge experiments using ground motion simulation. Geotech Test J 9:61-71

19. Mohammadi A, Qadimi A (2015) Characterizing the process of liquefaction initiation in Anzali shore sand through critical state soil mechanics. Soil Dyn Earthq Eng 77:152-163

20. ASTM D422-63 (2007) Standard test method for particle-size analysis of soils. ASTM International, West Conshohocken

21. Tsuchida $H$ (1970) Prediction and counter measure against the liquefaction in sand deposits. Seminar Abstract In: Port Harbour Research Institute, pp 3.1-3.33

22. ASTM D 2487 (2017) Standard practice for classification of soils for engineering purposes (Unified Soil Classification System) ASTM International West Conshohocken, PA, USA

23. Ishihara K (1993) Liquefaction and flow failure during earthquakes. Géotechnique 43:351-451. https://doi.org/10.1680/ geot.1993.43.3.351

24. Dobry R (1995) Centrifuge modeling of liquefaction effects during earthquakes. In: Proceedings of 1 st international conference on earthquake geotechnical engineering pp 1291-1324

25. Elgamal A, Zeghal M, Taboada V, Dobry R (1996) Analysis of site liquefaction and lateral spreading using centrifuge testing records. Soils Found 36:111-121

26. Zhang J, Cao J, Huang S (2018) Effects of initial shear stress and vibration frequency on the dynamic pore-water pressure of saturated sands. Adv Civ Eng 2018:1-13

27. Baziar MH, Shahnazari H, Sharafi H (2011) A laboratory study on the pore pressure generation model for Firouzkooh silty sands using hollow torsional test. Int J Civ Eng 9:126-134

28. Polito CP, Green RA, Lee J (2008) Pore pressure generation models for sands and silty soils subjected to cyclic loading. J Geotech Geoenviron Eng 134:1490-1500

29. Dobry R, Ladd RS, Yokel FY et al (1982) Prediction of pore water pressure build-up and liquefaction of sands during earthquakes by the cyclic strain method. National Bureau of Standards, Gaithersburg, $\mathrm{p} 176$

30. Seed HB, Martin PP, Lysmer J (1976) Pore-water pressure changes during soil liquefaction. J Geotech Geoenviron Eng 102:323-346

31. Konstadinou M, Georgiannou VN (2014) Prediction of pore water pressure generation leading to liquefaction under torsional cyclic loading. Soils Found 54:993-1005

32. Marasini N, Okamura M (2014) Liquefaction potential analysis and possible remedial measure for existing structure in Kathmandu Valley. Int J Landslide Environ 2:32-44

33. Zhao C, Koseki J, Miyashita Y (2017) Direct and indirect local deformations of sand in undrained cyclic triaxial tests by image analysis technique. In: Advances in Laboratory Testing and Modelling of Soils and Shales. Springer, Cham, pp 215-222

34. Kokusho T (1999) Water film in liquefied sand and its effect on lateral spread. J Geotech Geoenviron Eng 125:817-826

Publisher's Note Springer Nature remains neutral with regard to jurisdictional claims in published maps and institutional affiliations. 